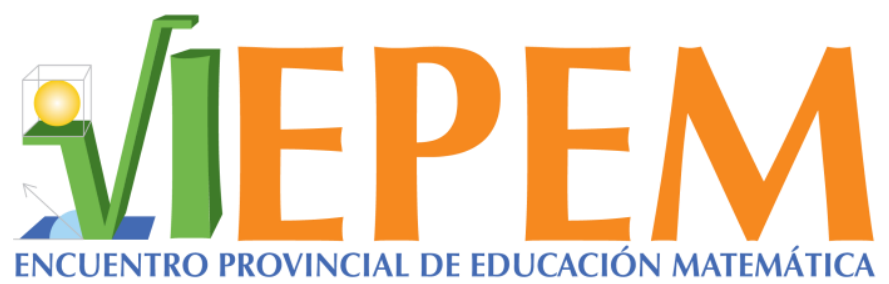

VI Encuentro Provincial de Educación Matemática.

27 al 29 de setiembre, 2017. Puntarenas, Costa Rica.

\title{
Mini MOOCs: una herramienta para la preparación del docente.
}

\author{
Óscar Salas-Huertas \\ oscar.salas.huertas@una.cr \\ Universidad Nacional \\ Costa Rica \\ Melvin Ramírez-Bogantes \\ meramirez@itcr.ac.cr \\ Instituto Tecnológico de Costa Rica \\ Costa Rica
}

\begin{abstract}
Resumen
Los programas de matemática vigentes en Costa Rica para la preparación de estudiantes de primaria y de secundaria representan un reto para los maestros y docentes en servicio. El tipo de metodología propuesto asume una mayor formación en aspectos propios de la Educación Matemática, por ejemplo, el dominio de las teorías de aprendizaje o las pautas para una adecuada mediación pedagógica. Desde este punto de vista, con esta actividad se busca reforzar los elementos claves que deben ser analizados al elegir una situación problema para organizar las lecciones según las sugerencias presentes en el currículo. Para lograr lo anterior, se recurre a plataformas disponibles al público interesado, denominadas Mini MOOCs, las cuales son de uso libre y gratuito, sin embargo, su impacto en los docentes del país no ha sido el esperado y por lo tanto, se pondrán en primer plano con la intención de que sean exploradas y extraer el potencial pedagógico y didáctico.
\end{abstract}

Palabras clave: Educación Matemática; Mediación pedagógica; Mini MOOCs

\section{Introducción}

Con la aprobación, en mayo del 2012, de los programas vigentes de matemática para primaria y secundaria, Costa Rica inició un cambio valiente y aceptó el reto de formar en esta disciplina individuos capaces de entender la importancia de los conceptos que aprenden y a su vez percibir la matemática como una herramienta poderosa para dar

Taller

\footnotetext{
Salas-Huertas, O. y Ramírez-Bogantes, M. (2017). Mini MOOCs: una herramienta para la preparación del docente. En Y. Morales-López, M. Picado, R. Gamboa, C. Martínez, M. Castillo y R. Hidalgo (Eds.), Memorias del VI Encuentro Provincial de Educación Matemática, Costa Rica, 2017 (pp. 35-37). Heredia: Universidad Nacional. ISBN: 978-9968-9661-5-3. DOI: http://dx.doi.org/10.15359/epem.6.8
} 
Mini MOOCs: una herramienta para la preparación del docente.

respuesta a múltiples interrogantes de la vida cotidiana, así como su papel en el desarrollo de nuevos conceptos que potencian el desarrollo científico y tecnológico de las naciones.

Como es predecible cuando se aspira a grandes cambios, los mismos van asociados a grandes retos, uno de ellos es superar o mitigar el cambio abrupto en la metodología de trabajo propuesta para avanzar con los contenidos y alcanzar las habilidades y competencias en cada nivel. La metodología propuesta considera dos momentos que están íntimamente ligados con la mediación pedagógica, estos son: el aprendizaje de los conocimientos y la movilización y aplicación de estos (MEP, 2012).

Para apoyar a los docentes en esta transición el MEP ha puesto a disposición de los profesionales una colección de Mini MOOCs (Massive Open Online Courses), los cuales "consisten en cursos gratuitos especializados en línea, dirigidos a un público masivo e impartidos por expertos en diversas áreas del conocimiento..." (Ramírez-Vega, 2015, p. $115)$.

\section{Objetivo del taller}

Fortalecer en los docentes las habilidades para generar en matemática situaciones problema que permitan introducir un nuevo contenido a través de la herramienta Mini MOOCs, según los lineamientos sugeridos en los actuales programas de Costa Rica.

\section{Metodología del taller}

Sesión 1: Bienvenida, descripción del taller, se establecen subgrupos de trabajo y se les asigna una situación problema presente en un Mini MOOC. Los facilitadores proporciona a cada grupo el material que considere pertinente para apoyar la solución del ejercicio. Se cierra con una breve exposición de cada subgrupo de la solución obtenida.

Sesión 2: Resumen de los alcances de la primera parte. Cada subgrupo completa las actividades pendientes del Mini MOOC asignado el día anterior. Cada subgrupos debe exponer a los participantes las vivencias más significativas y sus inquietudes sobre las actividades desarrolladas. Finalmente, cada subgrupo plantea actividades que puedan ser consideradas en un Mini MOOC.

\section{Resultados esperados}

Se espera que los docentes compartan sus experiencias en cuanto a beneficios y dificultades que surgen cuando se aplica la mediación pedagógica sugerida en los programas de matemática. Además, se espera que los docentes adquieran competencias para identificar situaciones problema apropiadas para introducir nuevos conceptos en el salón de clase y valoren el papel de los Mini MOOCs en este sentido. 
Mini MOOCs: una herramienta para la preparación del docente.

\section{Referencias}

Ministerio de Educación Pública. (2012). Programas de estudio de matemáticas. I y II Ciclo de la Educación Primaria, III Ciclo de Educación General Básica y Educación Diversificada. San José, Costa Rica: autor.

Ramírez-Vega, A. (2015). Nuevas tendencias de formación continua de educación matemática en Costa Rica: desarrollo e implementación de MOOCs. Cuadernos de Investigación y Formación en Educación Matemática, (13). Recuperado desde: https://revistas.ucr.ac.cr/index.php/cifem/article/view/19148.

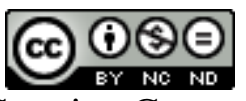

Esta obra está bajo una licencia de Creative Commons Reconocimiento-NoComercialSinObraDerivada 4.0 Internacional. 